\title{
No Linguistic Borders Ahead? Looking Beyond the Knocked-down Language Barrier
}

\section{Tomáš Svoboda Charles University, Prague}

The article deals with the concept of borders and barriers in considering scenarios where the linguistic barrier is eventually lifted by technology one day. It begins with reflections on the biblical narrative of the Tower of Babel as an ancient representation of the concept of linguistic barriers between language communities. It gives numerous examples of the uptake of this narrative, from Translation Studies, to project calls and the marketing statements of machine translation technology. In the following section, examples of existing technology are presented, which could be considered as a first generation of automatic translation/interpretation systems. In the main section, several trends are predicted for both the translators' profession and general economic/business/political/societal developments. The consequences are anticipated of a situation where ordinary cross-language communication will eventually have been almost fully taken over by automated systems. The article points to both the technology's positive potential and, by showing the eventual risks involved, it equally rejects an attitude of the technology's uncritical uptake. The article closes by pointing to the ethical dimension of machine translation systems linked with their types of uses and the choices reserved for their users. 